\title{
Chicken innate immune response to oral infection with Salmonella enterica serovar Enteritidis
}

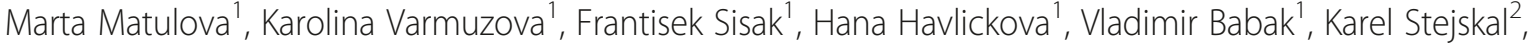 \\ Zbynek Zdrahal ${ }^{2}$ and Ivan Rychlik ${ }^{1 *}$
}

\begin{abstract}
The characterization of the immune response of chickens to Salmonella infection is usually limited to the quantification of expression of genes coding for cytokines, chemokines or antimicrobial peptides. However, processes occurring in the cecum of infected chickens are likely to be much more diverse. In this study we have therefore characterized the transcriptome and proteome in the chicken cecum after infection with Salmonella Enteritidis. Using a combination of 454 pyrosequencing, protein mass spectrometry and quantitative real-time PCR, we identified 48 down- and 56 up-regulated chicken genes after Salmonella Enteritidis infection. The most inducible gene was that coding for MMP7, exhibiting a 5952 fold induction 9 days post-infection. An induction of greater than 100 fold was observed for IgG, IRG1, SAA, ExFABP, IL-22, TRAP6, MRP126, IFNY, iNOS, ES1, IL-13, LYG2, IFIT5, IL-17, AVD, AH221 and SERPIN B. Since prostaglandin D2 synthase was upregulated and degrading hydroxyprostaglandin dehydrogenase was downregulated after the infection, prostaglandin must accumulate in the cecum of chickens infected with Salmonella Enteritidis. Finally, above mentioned signaling was dependent on the presence of a SPI1-encoded type III secretion system in Salmonella Enteritidis. The inflammation lasted for 2 weeks after which time the expression of the "inflammatory" genes returned back to basal levels and, instead, the expression of IgA and IgG increased. This points to an important role for immunoglobulins in the restoration of homeostasis in the cecum after infection.
\end{abstract}

\section{Introduction}

Salmonella enterica is one of the most frequent causative agents of human gastrointestinal disorders with the major sources of $S$. enterica isolates for the human population originating from farm animal production, pigs and poultry in particular. Poultry flocks are reservoirs of S. enterica serovar Enteritidis (Salmonella Enteritidis), the serovar whose incidence in the human population has increased considerably since the beginning of the 1990s [1,2]. As poultry is a major source of Salmonella Enteritidis for humans, it is believed that the measures applied in chicken egg production, which will lead to a decrease of Salmonella Enteritidis prevalence, will also affect the incidence of salmonellosis in the human population. This is why a program aimed at decreasing the prevalence of Salmonella in poultry flocks is currently implemented in the EU [1,2].

\footnotetext{
* Correspondence: rychlik@vri.cz

'Veterinary Research Institute, Hudcova 70, Brno 621 00, Czech Republic Full list of author information is available at the end of the article
}

Despite the absence of gross clinical signs, chickens respond to oral infection with non-typhoid serovars of S. enterica by moderate inflammation in the cecum associated with heterophil and monocyte/macrophage infiltration into the cecal mucosa. In agreement with this, the expression of proinflammatory cytokines such as IL$1 \beta$, IL-6, IL-17, IL-22 and IFNy together with iNOS is increased in the cecum of infected chickens [3-5]. The production of the above cytokines is either induced in epithelial cells and resident phagocytes, or is affected by infiltrating phagocytes or lymphocytes [6]. However, this is clearly a simplified and an incomplete view of the chicken's response to Salmonella infection as, for example, in mice genes not involved in cytokine signaling, e.g., Lcn2, are induced in the small intestine upon infection with Salmonella enterica serovar Typhimurium [7]. Moreover, by using pyrosequencing of RNA transcripts in the spleen of chickens intravenously infected with Salmonella Enteritidis, we have identified many new genes which have not been associated with Salmonella
Ciomed Central

(c) 2013 Matulova et al.; licensee BioMed Central Ltd. This is an Open Access article distributed under the terms of the Creative Commons Attribution License (http://creativecommons.org/licenses/by/2.0), which permits unrestricted use, distribution, and reproduction in any medium, provided the original work is properly cited. 
infection in chickens so far [6]. When we tested whether these genes were also induced in the cecum, 14 of them were indeed upregulated in the cecum of orally infected chickens, one of them being a functional homologue of murine Lcn2 [6].

This prompted us to perform a genome-wide characterization of the chicken response in the cecum to oral infection with Salmonella Enteritidis. Unlike our previous paper on expression in the spleen [6], we characterized both the transcriptome and proteome by pyrosequencing and protein mass spectrometry, respectively, and the initial results were verified by real time RT-PCR. The combination of both experimental procedures allowed us to characterize the events occurring in the chicken cecum at quite a detailed level and define the individual steps in the chicken's innate immune response to oral infection with Salmonella Enteritidis.

\section{Material and methods \\ Ethics statement}

The handling of animals in the study was performed in accordance with current Czech legislation (Animal protection and welfare Act No. 246/1992 Coll. of the Government of the Czech Republic). The specific experiments were approved by the Ethics Committee of the Veterinary Research Institute (permit number 48/2010) followed by the Committee for Animal Welfare of the Ministry of Agriculture of the Czech Republic (permit number MZe 1226).

\section{Experimental animals, sample collection and pyrosequencing}

The ceca of 6 ISA Brown chickens were used for simultaneous RNA and protein isolation. Three of these chickens were infected orally with $10^{7} \mathrm{CFU}$ of Salmonella Enteritidis on the day of hatching and sacrificed 4 days later while the other 3 represented non-inoculated 5-day-old control chickens. Approximately $30 \mathrm{mg}$ of cecum was collected from each chicken during necropsy and immediately placed into RNALater (Qiagen). The samples were then mixed with TRI Reagent and processed according to the manufacturer's recommendations (MRC). In brief, after centrifugation for $15 \mathrm{~min}$ at $14000 \mathrm{~g}$, the upper phase containing RNA was purified with an RNeasy Mini Kit (Qiagen) followed by an mRNA Isolation Kit (Roche) to enrich the RNA preparation for mRNA species. Proteins captured in the lower phase were precipitated from the TRI Reagent with 3 volumes of acetone, washed with guanidine hydrochloride (0.3 M)/ethanol (95\%)/glycerol (2.5\%) wash solution and dried in a SpeedVac.

Because of the cost of pyrosequencing (see below), only pooled RNA/cDNA samples of 3 infected and 3 noninfected chickens were processed, respectively. For protein mass spectrometry, the samples from the 3 infected and 3 non-infected chickens were randomly grouped into 3 pairs and analyzed.

To verify the results from mRNA pyrosequencing and protein mass spectrometry, cDNA samples kept at $-20^{\circ} \mathrm{C}$ from our previous experiments were used in real-time PCR quantification [6]. These samples originated from the cecum of orally infected 5-day-old chickens and included samples from 2 independent experiments, each performed with 4 infected and 4 non-infected chickens.

\section{Time-dependent gene expression in chicken cecum after Salmonella Enteritidis infection}

A time-dependent characterization of gene expression in the cecum was performed with 64 chickens which were orally infected with $10^{7} \mathrm{CFU}$ of Salmonella Enteritidis on the day of hatching and sacrificed on days $2,3,4,5,6,7,8$, $9,10,11,12,15,18,22,25$ and 29 of life, 4 chickens on each day. On day 1 and at all additional sampling times, 4 non-infected control chickens were sacrificed as well. From each of the chickens, mRNA from cecum was purified, reverse transcribed into cDNA and subjected to real time PCR as described below. To verify the infection, Salmonella Enteritidis counts in the liver, spleen and cecum were determined by serial dilutions of tissue homogenates in peptone water and plating on XLD agar, as described previously [8].

\section{Influence of Salmonella Enteritidis SPI1 on the inflammation in chicken cecum}

In the last experiment, we infected 8 newly hatched chickens with the wild-type Salmonella Enteritidis or an isogenic SPI1 mutant of Salmonella Enteritidis [9], and sacrificed them 4 days later. Four non-infected control chickens sacrificed on day 5 of life were included as well. During necropsy, cecal wall samples were placed in RNALater followed by RNA purification using an RNeasy Mini Kit (Qiagen).

\section{4 pyrosequencing}

cDNA libraries from the cecal samples were prepared with the GS Rapid Library Preparation Kit (Roche) and approx. 2 molecules of cDNA per bead were used in emulsion PCR. All steps of the cDNA library preparation were performed with the GS Junior Titanium series kits according to the manufacturer's instructions (Roche). The pyrosequencing was performed with the GS Junior 454 sequencer (Roche) and each sample was sequenced in a separate sequencing run.

Data analysis after pyrosequencing was performed exactly as described previously except for the fact that the total chicken transcriptome was generated using the sequences from the previous pyrosequencing of the spleen samples enriched for the two new sequencing runs from 
the cecum [6]. Transcripts predicted as differentially expressed after Salmonella Enteritidis infection included those for which we identified at least a 12-fold up- or down-regulation regardless of the number of reads available, or a 6-fold up- or down-regulation with 25 independent reads available, or a 3-fold up- or down-regulation with at least 50 independent reads.

\section{Protein identification and quantification}

Protein pellets from 3 infected and 3 non-infected chickens were processed by the filter-aided sample preparation (FASP) method [10]. Pellets were diluted in $100 \mu \mathrm{L}$ of $4 \%$ SDS, 0.1M DTT, 0.1M Tris- $\mathrm{HCl} \mathrm{pH} 7.6$, incubated for 5 min at $95^{\circ} \mathrm{C}$, cooled to room temperature and centrifuged at $20000 \times g$ for $15 \mathrm{~min}$ at $20^{\circ} \mathrm{C}$. The supernatant was collected and mixed with $8 \mathrm{M}$ UA buffer ( $8 \mathrm{M}$ urea in $100 \mathrm{mM}$ Tris- $\mathrm{HCl}, \mathrm{pH} 8.5$ ) at a ratio of $1: 13$, loaded onto the Vivacon 500 device with MWCO $10 \mathrm{kDa}$ (Sartorius Stedim Biotech) and centrifuged at $14000 \times g$ for $30 \mathrm{~min}$ at $20^{\circ} \mathrm{C}$. The retained proteins were washed with $400 \mu \mathrm{L}$ UA buffer. The final protein concentrates kept in the Vivacon 500 device were mixed with $100 \mu \mathrm{L}$ of UA buffer containing $50 \mathrm{mM}$ iodoacetamide and incubated in the dark for $30 \mathrm{~min}$. After an additional centrifugation, the samples were washed three times with $400 \mu \mathrm{L}$ UA buffer and three times with $200 \mu \mathrm{L}$ of $50 \mathrm{mM} \mathrm{NaHCO}$. Trypsin (sequencing grade, Promega) was added onto the filter and the mixture was incubated for $2 \mathrm{~h}$ at $40^{\circ} \mathrm{C}$. The tryptic peptides were finally eluted by centrifugation followed by two additional elutions with $50 \mu \mathrm{L}$ of $50 \mathrm{mM} \mathrm{NaHCO}$. The concentration of the eluted tryptic peptides was determined by UV-spectrometry using an extinction coefficient of 1.1 for $0.1 \%(\mathrm{~g} / \mathrm{L})$ solution at $280 \mathrm{~nm}$ [11].

Tryptic peptides prepared by FASP were labeled as described elsewhere [12]. The concentration of each sample was adjusted with $50 \mathrm{mM} \mathrm{NaHCO}$ to a final concentration of $1.5 \mu \mathrm{g} / \mu \mathrm{L}$ and $10 \mu \mathrm{L}$ of peptide solution was mixed with $90 \mu \mathrm{L} 0.1 \mathrm{M}$ sodium acetate buffer $\mathrm{pH} 6.0$ followed by the addition of $20 \mu \mathrm{L} 4 \%$ formaldehyde- $\mathrm{d}_{0}$ (samples from the non-infected chickens) or formaldehyde- $\mathrm{d}_{2}$ (samples from the infected chickens) and $20 \mu \mathrm{L} 1 \mathrm{M} \mathrm{NaBCNH}$. After $10 \mathrm{~min}$, the reactions were stopped by adding $20 \mu \mathrm{L}$ $4 \% \mathrm{NH}_{3}$ and the samples were randomly grouped into three pairs, each consisting of a sample from the infected and non-infected chicken.

LC-MS/MS analyses of dimethylated peptide mixture were done using RSLCnano system connected to Orbitrap Elite hybrid spectrometer (Thermo Fisher Scientific). Prior to LC separation, tryptic digests were concentrated and desalted using a trapping column $(100 \mu \mathrm{m} \times 30 \mathrm{~mm})$ filled with $3.5-\mu \mathrm{m}$ X-Bridge $\mathrm{BEH}$ 130 C18 sorbent (Waters). After trapping the column was washed with $0.1 \%$ FA, the peptides were eluted (flow $300 \mathrm{nl} / \mathrm{min}$ ) onto an Acclaim Pepmap100 C18 column $(2 \mu \mathrm{m}$ particles, $75 \mu \mathrm{m} \times 250 \mathrm{~mm}$; Thermo Fisher Scientific) using the following gradient program (mobile phase A: $0.1 \%$ FA in water; mobile phase B: ACN:methanol:2,2,2-trifluoroethanol (6:3:1; v/v/v) containing $0.1 \%$ FA). The gradient elution started at $2 \%$ of mobile phase B and increased from $2 \%$ to $45 \%$ during the first $90 \mathrm{~min}$, then increased linearly to $95 \%$ of mobile phase $B$ in the next $5 \mathrm{~min}$ and remained at this state for the next $15 \mathrm{~min}$. The analytical column outlet was directly connected to the Nanospray Flex Ion Source (Thermo Fisher Scientific).

MS data were acquired in a data-dependent strategy selecting the top 20 precursors based on the precursor abundance in the survey scan $(350-1700 \mathrm{~m} / \mathrm{z})$. The resolution of the survey scan was 120000 (at $400 \mathrm{~m} / \mathrm{z}$ ) with a target value of $1 \times 10^{6}$ ions, one microscan and maximum injection time of $200 \mathrm{~ms}$. Low resolution CID MS/MS spectra were acquired with a target value of 10000 ions in rapid CID scan mode with the $\mathrm{m} / \mathrm{z}$ range adjusted according to the actual precursor mass and charge. MS/ MS acquisition in the linear ion trap was carried out in parallel with the survey scan in the Orbitrap analyzer by using the preview mode. Dynamic exclusion was enabled for $45 \mathrm{~s}$ after one MS/MS spectra acquisition and early expiration was disabled. The isolation window for MS/MS fragmentation was set to $2 \mathrm{~m} / \mathrm{z}$.

The analysis of the mass spectrometric RAW data files was carried out using the Proteome Discoverer software (version 1.3) with an in-house Mascot and Sequest search engine utilization. Mascot and Sequest MS/MS ion searches were performed against the NCBI protein database (taxonomy Gallus gallus). Mass tolerance for peptides and MS/MS fragments were $5 \mathrm{ppm}$ and $0.5 \mathrm{Da}$, respectively. Carbamidomethylation (C), dimethylation (N-term and $\mathrm{K}$ ) as fixed modification, oxidation (M) as optional modification and none enzyme miss cleavage were set for all searches. Percolator was used for postprocessing of Mascot search results. Peptides with a false discovery rate (FDR; q-value) $<1 \%$ and at least 3 significant peptides were considered.

\section{Real-time PCR verification of the pyrosequencing data}

Real-time PCR was used for the verification of both pyrosequencing and proteomic data. Primers for the quantification of expression real-time PCR were designed using Primer3 software [13] (for primer sequences see Additional file 1). First we used the same cDNAs as in the pyrosequencing reactions followed by an additional $2 \times 4$ samples from our previous study [6]. After such a screening we reduced the number of tested genes to those in which the real-time PCR confirmed the results from the pyrosequencing or proteomics and exhibited fold up- or downregulation greater than 4 . Using the reduced set of real-time PCRs, we finally determined the gene expression 
in i) the time-dependent study and ii) after the infection with the SPI1 mutant.

Real-time PCR was performed in $3 \mu \mathrm{L}$ volumes in 384well microplates using QuantiTect SYBR Green PCR Master Mix (Qiagen) and a Nanodrop II Stage pipetting station (Innovadyne) for PCR mix dispensing. Real-time PCR was performed using a LightCycler II (Roche) with an initial denaturation at $95^{\circ} \mathrm{C}$ for $15 \mathrm{~min}$ followed by 40 cycles of $95^{\circ} \mathrm{C}$ for $20 \mathrm{~s}, 60^{\circ} \mathrm{C}$ for $30 \mathrm{~s}$ and $72^{\circ} \mathrm{C}$ for $30 \mathrm{~s}$. Each sample was subjected to real-time PCR in duplicate and the mean $\mathrm{Ct}$ value of the duplicates was used for subsequent analysis. The Ct values of the genes of interest were normalized $(\Delta \mathrm{Ct})$ to an average $\mathrm{Ct}$ value of three house-keeping genes (GAPDH, TBP and UB) and the relative expression of each gene of interest was calculated as $2^{-\Delta C t}$. These expression levels were used for data analysis and are presented in the tables and figures as average $\pm \mathrm{SD}$. As an alternative, the fold upregulation (i.e. $2^{-\Delta \Delta C t}$ ) calculated as the ratio of averages of infected to non-infected samples is presented. However, also in this case, the significance of such upregulations was calculated by comparing the expressions, i.e. the $2^{-\Delta \mathrm{Ct}}$ values of the individual samples.

\section{Northern blot analysis}

The expression of selected genes (MMP7, ExFABP, AQP8 and $\mathrm{ADH} 1$ ) was confirmed also by northern blotting. The same mRNAs of 6 ISA Brown chickens which was used for pyrosequencing was electrophoretically separated in formaldehyde denaturing agarose gel and vacuum blotted onto a nylon membrane as described previously [14]. Probes for the northern hybridization were prepared by PCR followed by purification using a PCR Purification kit from Qiagen according to the manufacturer's instructions. Probe labeling, hybridization, posthybridization washes and signal development were performed with an AlkPhos labelling kit according to the manufacturer's instructions (GE Healthcare). A GAPDH specific probe was used as a control of loading the same amount of RNA.

\section{Statistics}

Heat maps were generated using software available elsewhere [15]. The statistical significance of the gene expression 4 days post infection were determined by a $t$-test. PCA have been calculated in logarithmically transformed data ignoring the absolute transcription levels and following the transcriptional profiles only. All the statistical calculations have been done using Statistica 9.1 software (StatSoft, Inc., Tulsa, OK, USA) and differences with $p<0.05$ were considered as significant.

\section{Results}

\section{Pyrosequencing}

Pyrosequencing resulted in 96459 reads (43 $257673 \mathrm{nt}$ of RNA sequence) when pooled cDNA from the cecum of the non-infected chicken was sequenced and 43458 reads (18 $403094 \mathrm{nt}$ of RNA sequence) when pooled cDNA from the cecum of the chicken after Salmonella Enteritidis infection was sequenced. Combining these 2 samples together with the data sets from our previous study on the expression in chicken spleen [6], the de novo assembly resulted in the identification of 13063 different chicken transcripts. After applying all the quality selective criteria, 35457 reads from the cecum of the non-infected chicken and 29586 reads from the cecum of the infected chicken were finally included in the quantification of gene expression and we predicted that 54 genes might be downregulated and 78 genes upregulated in the cecum after Salmonella Enteritidis infection (Additional file 1).

\section{Mass spectrometry}

Using LC-MS/MS analysis, 4062 protein groups containing at least 1 peptide with a significant score were identified in an experimental set of 3 infected and 3 non-infected chicken samples. There were 806 protein groups which were quantified as having at least 3 peptides with a significant score and these were selected for further evaluation. At least a twofold down- or up-regulation was observed for 21 and 57 proteins, respectively (Additional file 1).

\section{Real-time PCR}

In the next experiment we verified the expression of 195 genes predicted by either 454 pyrosequencing or mass spectrometry as responsive to Salmonella Enteritidis infection by real-time PCR. The predicted expression profiles were confirmed for 104 of them, with 48 genes confirmed as significantly downregulated and 56 as significantly upregulated (Additional file 1).

\section{Northern blot analysis}

Since all the above mentioned protocols can be considered as indirect, northern blot analysis was performed for 2 genes (AQP8, ADH1B) that were downregulated after infection and 2 genes (ExFABP, MMP7) that were upregulated after infection using samples originating from those used for pyrosequencing and mass spectrometry. The northern blot analysis confirmed the results of real-time PCR in all 4 cases (Figure 1).

\section{Time-dependent expression in the cecum of Salmonella Enteritidis infected chickens}

In previous experiments, gene expression was determined only at a single time point when the expression of some of the genes could have been already in decline whilst the expression of the others could still have been on the increase. In the next experiment we therefore characterized the chicken's response to Salmonella Enteritidis infection in a time-dependent study. Salmonella Enteritidis counts 
in the cecum reached a maximum as early as $24 \mathrm{~h}$ after oral infection and then gradually decreased. The liver and spleen became highly colonized $48 \mathrm{~h}$ after oral infection. Salmonella Enteritidis counts in the liver and spleen then remained relatively constant until day 12 , from which point on, a gradual decrease in Salmonella Enteritidis counts was observed (Figure 2).

For monitoring time-dependent gene expression in the ceca we selected only 43 different genes. These included 32 genes identified in this study which were up- or down-regulated more than 4-fold. In addition, we also included 7 genes (avidin, trappin-6, chemokine AH221, IRG1, serum amyloid A, IgG, IgA) which we identified previously as responding to Salmonella Enteritidis infection [6] and 4 genes (IL-1 $\beta$, IL-17, IL-22, IFN $\gamma$ ) which are commonly used as markers of inflammation [3-5]. There were 3 groups among the genes that showed induction after Salmonella Enteritidis infection which differed in the times of their maximal expression. The first group of genes was characterized by peak expression 2, 3 or 4 days after infection. This group included genes coding for lysozyme G2, IL-8, IL-17, serum amyloid A, trappin-6, ExFABP, ES1, IL-1 $\beta$ and prostaglandin synthase (Figure 2). The second group of genes exhibited a prolonged, high level expression and these included genes coding for STAT1, STAT3, epithelial stromal factor, arginine-succinate lyase, hematopoietic lineage protein, CD18, C3 complement protein and IFNץ. The last

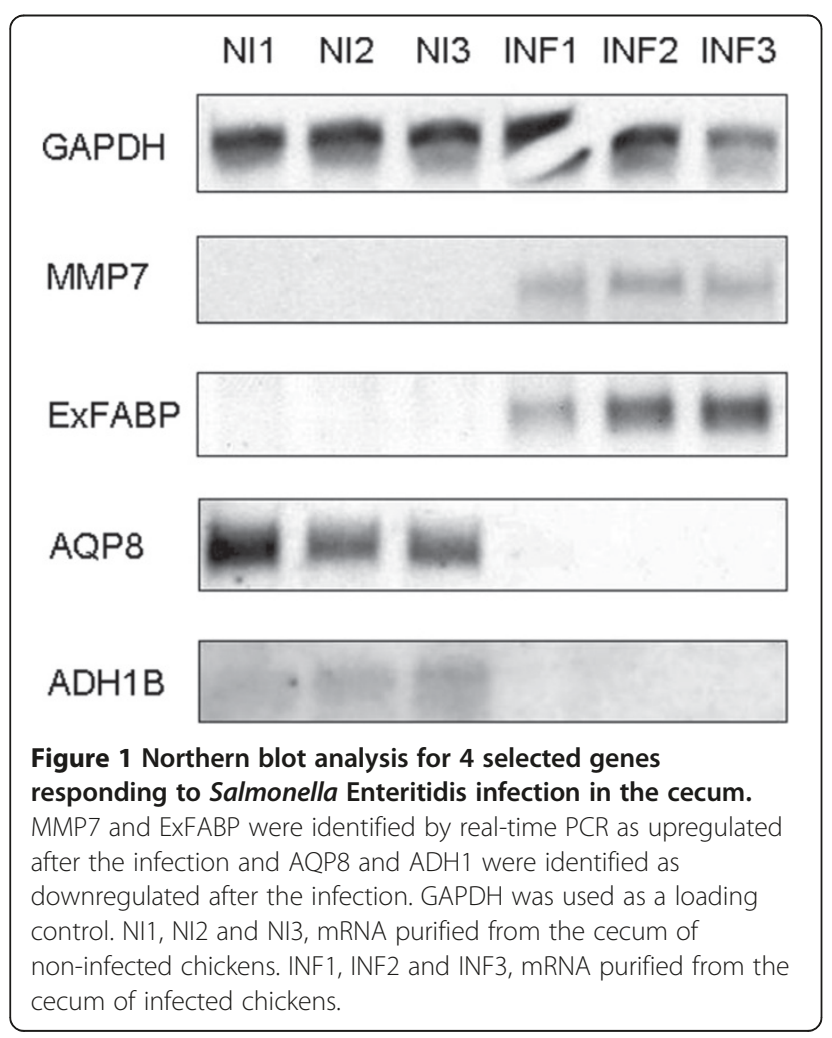

group of genes upregulated after Salmonella Enteritidis infection was formed by those coding for the constant part of the IgG and IgA heavy chains. Their expression increased above background levels for the first time on day 7 (IgG) and day 15 (IgA), respectively, and reached their maximal expression on day 22. Except for the immunoglobulin encoding genes, the expression of all the upregulated genes decreased between days 12 and 15 nearly to the levels observed in the non-infected chickens (Figure 3).

The genes which were downregulated in response to Salmonella Enteritidis infection exhibited such a profile from $48 \mathrm{~h}$ after the infection and their suppression lasted until day 15, from which time their expression began to recover (Figure 3).

\section{Contribution of individual genes to total expression in chicken cecum}

Since the heat map in Figure 3 was normalized for each individual gene according to its minimal and maximal expression, it did not provide a realistic view of how individual genes contribute to the total gene expression in the cecum. Gene expression in the cecum of the non-infected chickens was dominated by aquaporin 8, alcoholdehydrogenase, fatty acid binding protein 1 , retinal dehydrogenase, uncharacterized oxidoreductase and calbindin 1 (Table 1), i.e., the genes which were suppressed after Salmonella Enteritidis infection. Two days after infection, the above mentioned genes therefore formed a clear "expression" minority and they reached their minimal expression on day 5 when the transcription in the cecum was

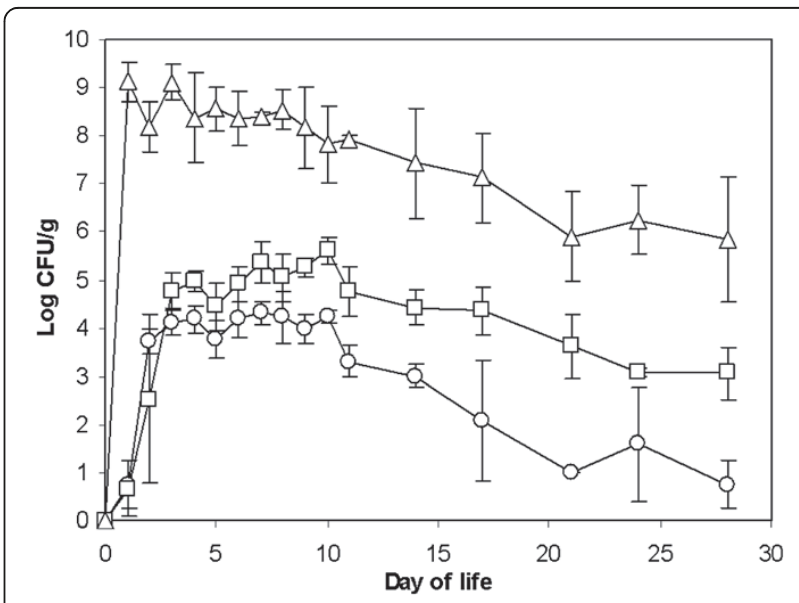

Figure 2 Salmonella Enteritidis colonization of the cecum, liver and spleen after oral infection at the day of hatching. The cecum (triangles) was colonized as early as at the first sampling time, $24 \mathrm{~h}$ after the infection. Colonization of the liver (circles) and spleen (squares) required an additional $24 \mathrm{~h}$ and these organs became highly colonized from $48 \mathrm{~h}$ after the infection. The liver and spleen remained colonized at constant counts until day 12 of life and thereafter a gradual decrease in Salmonella Enteritidis counts was observed. 


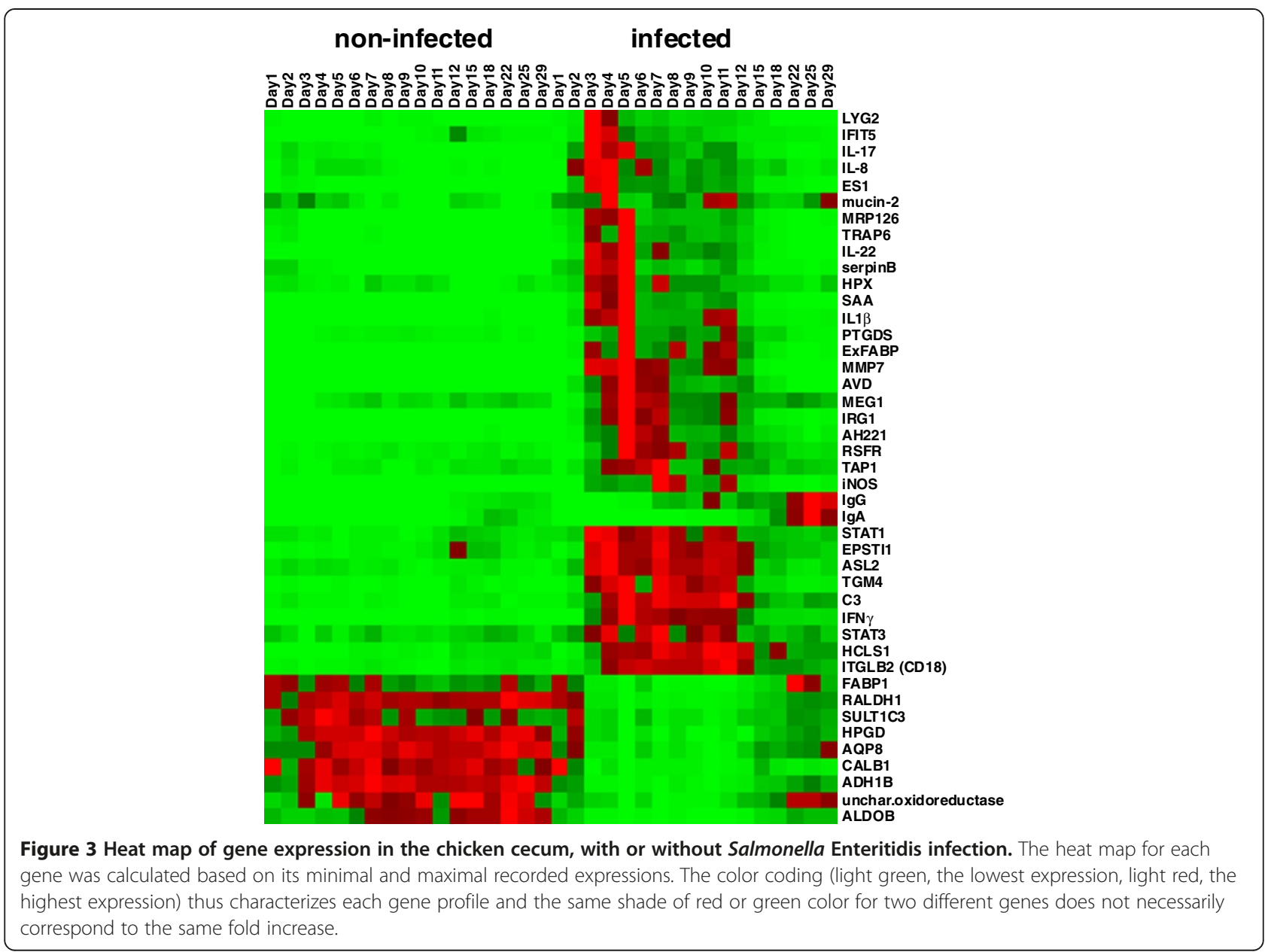

dominated by ES1 protein, avidin, AH221 chemokine, ExFABP and serum amyloid A (Table 1). In addition, high expression of IgG and IgA was recorded from day 15 reaching its maximum on day 22 and 25, respectively (Table 1).

\section{Identification of genes with the highest up- or down- regulation}

The absolute expression of each of the 43 tested genes, however, need not correlate with their fold induction after Salmonella Enteritidis infection. The most downregulated gene was that coding for aquaporin 8 with a downregulation as low as 97 fold observed 5 days postinfection. The suppression of the remaining genes, though lower than that of aquaporin 8 , were quite similar and ranged from 18 (SULT1C3) to 75 (ADH1B) fold (Table 1). The gene with the highest induction was that encoding matrix metalloproteinase MMP7 whose expression increased more than 3 logs with the highest 5952 fold upregulation observed 9 days after the infection. Upregulation of an additional 17 genes exceeded a factor of 100 and upregulation of the remaining 16 genes was below a factor of 100 (Table 1).

\section{PCA of individual chickens based on their gene expression in the cecum}

The time-dependent experiment was performed with 132 chickens, samples of which were subjected to 43 gene characteristics which resulted in a dataset with 5940 values. This dataset was next used for the clustering of each individual chicken according to its expression profile. PCA clustering of the expression profiles observed in individual chickens confirmed and further extended previous analyses. The gene expression gradually changed in time also in the cecum of the non-infected chickens (Figure 4). Salmonella Enteritidis infection on the day of hatching resulted in a minor response in the proceeding $24 \mathrm{~h}$ and the 2-day-old infected chickens still clustered close to the non-infected age-matched controls. However, from 2 days post infection, the expression in the cecum clustered the infected chickens separately from the non-infected controls. PCA also indicated a gradual re-joining of the infected chickens with the non-infected ones from day 15 
Table 1 List of genes up- or down-regulated in the chicken cecum after infection with Salmonella Enteritidis arranged according to their maximal fold up- or down-regulation after infection with wild type Salmonella Enteritidis (column max fold SE wt)

\begin{tabular}{|c|c|c|c|c|c|c|c|}
\hline Gene & Max $\mathrm{NI}^{\#}$ & Min NI & Max SE & Min SE & $\begin{array}{r}\text { Max fold } \\
\text { SE wt } \$\end{array}$ & $\begin{array}{c}\text { WT fold } \\
4 \mathrm{dpi}^{\&}\end{array}$ & $\begin{array}{r}\text { SPI1 fold } \\
4 \mathrm{dpi}^{\&} \\
\end{array}$ \\
\hline MMP7 & $0.078(12)$ & $0.001(29)$ & $8.98(5)$ & $0.001(1)$ & $5952(10)$ & 1578 & 35.2 \\
\hline $\lg G$ & $5.02(25)$ & $0.006(2)$ & $36.7(25)$ & $0.009(1)$ & $442(10)$ & 33.2 & 1.63 \\
\hline IRG1 & $0.005(2)$ & $0.001(11)$ & $0.69(5)$ & $0.003(1)$ & $425(7)$ & 222 & 4.61 \\
\hline SAA & $0.25(2)$ & $0.018(9)$ & $15.9(5)$ & $0.065(1)$ & $382(5)$ & 106 & 5.02 \\
\hline ExFABP & $1.06(18)$ & $0.150(9)$ & $75.3(5)$ & $0.369(1)$ & $366(5)$ & 56.8 & 4.80 \\
\hline IL-22 & 0.005 (12) & $0.0004(10)$ & $0.39(5)$ & $0.001(1)$ & 355 (10) & 46.9 & 2.69 \\
\hline TRAP6 & $0.39(2)$ & $0.011(15)$ & $5.76(5)$ & $0.029(25)$ & $194(5)$ & 37.3 & 2.19 \\
\hline MRP126 & $0.16(2)$ & $0.003(29)$ & $1.96(5)$ & $0.018(25)$ & $194(11)$ & 14.3 & 2.11 \\
\hline $\mathrm{IFNY}$ & $0.001(18)$ & $0.0001(11)$ & 0.035 & $0.0002(1)$ & $183(11)$ & 62.8 & 1.39 \\
\hline $\operatorname{IL} 1 \beta$ & 0.018 (18) & $0.002(1)$ & $0.74(5)$ & $0.002(1)$ & $134(3)$ & 29.1 & 1.59 \\
\hline iNOS & $0.019(1)$ & $0.008(9)$ & $1.99(7)$ & $0.019(1)$ & $151(7)$ & 47.9 & 2.84 \\
\hline ES1 & $2.06(2)$ & 0.055 (29) & $39.6(4)$ & $0.178(1)$ & $139(11)$ & 17.4 & 2.21 \\
\hline LYG2 & $0.26(7)$ & $0.015(22)$ & $5.82(3)$ & $0.031(25)$ & $130(3)$ & 24.5 & 3.09 \\
\hline IFIT5 & $0.56(12)$ & $0.003(1)$ & $1.28(3)$ & $0.003(1)$ & $124(3)$ & 42.4 & 2.62 \\
\hline IL-17 & $0.014(2)$ & $0.0003(29)$ & 0.095 (3) & $0.001(22)$ & $120(11)$ & 6.72 & 1.12 \\
\hline AVD & $2.07(2)$ & $0.20(29)$ & $103.1(5)$ & $0.207(1)$ & $116(9)$ & 29.6 & 3.24 \\
\hline $\mathrm{AH} 221$ & $0.73(18)$ & $0.12(1)$ & $23.4(5)$ & $0.119(1)$ & $108(5)$ & 42.9 & 1.90 \\
\hline serpin B & $0.096(2)$ & $0.002(29)$ & $0.54(5)$ & $0.009(25)$ & $101(5)$ & 18.4 & 1.45 \\
\hline TGM4 & $0.092(12)$ & $0.005(6)$ & $0.92(5)$ & $0.014(1)$ & $68(6)$ & 30.4 & 1.99 \\
\hline $\lg A$ & $6.04(18)$ & $0.003(2)$ & $23.4(22)$ & 0.005 (3) & 49 (3) & 16.6 & 1.86 \\
\hline EPSTI1 & $0.39(12)$ & $0.015(1)$ & $0.74(4)$ & $0.015(1)$ & $33(3)$ & 12.9 & 1.40 \\
\hline IL-8 & $0.15(6)$ & $0.004(29)$ & $1.08(4)$ & $0.007(1)$ & $31(11)$ & 4.54 & 1.24 \\
\hline C3 & $0.092(2)$ & $0.019(10)$ & $0.78(11)$ & $0.044(1)$ & $29(10)$ & 9.7 & 1.53 \\
\hline mucin-2 & $0.37(3)$ & $0.018(9)$ & $0.77(4)$ & $0.124(6)$ & $20(11)$ & 1.01 & 0.94 \\
\hline PTGDS & $1.50(9)$ & $0.12(1)$ & $17.8(5)$ & $0.117(1)$ & $19(5)$ & 14.9 & 1.70 \\
\hline ITGLB2 (CD18) & $0.084(22)$ & $0.031(3)$ & $0.46(11)$ & $0.045(18)$ & $14(11)$ & 9.40 & 1.04 \\
\hline $\mathrm{HPX}$ & $0.19(7)$ & $0.025(12)$ & $0.91(5)$ & $0.071(25)$ & $13(5)$ & 5.06 & 1.10 \\
\hline ASL2 & $0.46(2)$ & $0.15(29)$ & $2.26(4)$ & $0.289(1)$ & $11(10)$ & 3.74 & 1.17 \\
\hline HCLS1 & $0.25(29)$ & $0.088(5)$ & $1.11(10)$ & $0.107(1)$ & $10(11)$ & 5.41 & 1.13 \\
\hline TAP1 & $0.443(22)$ & $0.087(3)$ & $2.26(7)$ & $0.104(1)$ & $10(4)$ & 13.7 & 1.37 \\
\hline STAT1 & $0.31(12)$ & $0.059(9)$ & $1.03(3)$ & $0.164(1)$ & $9.2(9)$ & 8.61 & 1.25 \\
\hline RSFR & $0.96(8)$ & $0.40(1)$ & $7.19(5)$ & $0.397(1)$ & $9.1(5)$ & 6.41 & 1.19 \\
\hline MEG1 & $0.49(22)$ & $0.090(3)$ & $1.80(5)$ & $0.115(1)$ & $7.0(5)$ & 8.22 & 0.92 \\
\hline STAT3 & $0.64(18)$ & $0.37(3)$ & $1.28(7)$ & $0.537(5)$ & $2.2(3)$ & 2.91 & 1.00 \\
\hline AQP8 & $33.4(22)$ & $14.9(1)$ & $17.6(25)$ & $0.290(5)$ & $97(5)$ & 18.90 & 1.80 \\
\hline $\mathrm{ADH} 1 \mathrm{~B}$ & $9.46(7)$ & $3.42(2)$ & $4.66(25)$ & $0.100(5)$ & $75(5)$ & 16.23 & 1.55 \\
\hline FABP1 & $6.11(22)$ & $1.98(11)$ & $8.48(22)$ & $0.119(5)$ & $47(5)$ & 5.49 & 1.32 \\
\hline RALDH1 & $18.6(22)$ & $9.17(2)$ & $12.6(1)$ & $0.404(5)$ & $39(5)$ & 12.87 & 1.43 \\
\hline
\end{tabular}


Table 1 List of genes up- or down-regulated in the chicken cecum after infection with Salmonella Enteritidis arranged according to their maximal fold up- or down-regulation after infection with wild type Salmonella Enteritidis (column max fold SE wt) (Continued)

\begin{tabular}{|c|c|c|c|c|c|c|c|}
\hline unchar. oxidoreductase & $5.84(12)$ & $0.80(4)$ & $4.25(22)$ & $0.192(5)$ & $28(5)$ & 7.14 & 1.26 \\
\hline CALB1 & $24.2(1)$ & $8.20(2)$ & $24.2(1)$ & 0.785 (5) & $19(5)$ & 13.78 & .88 \\
\hline ALDOB & $1.66(22)$ & $0.32(2)$ & $0.58(22)$ & $0.037(5)$ & 19 (10) & 6.71 & 99 \\
\hline SULT1C3 & 21.9 (4) & 7.20 (29) & $16.0(1)$ & $1.081(5)$ & $18(5)$ & 8.04 & 86 \\
\hline HPGD & $4.62(7)$ & $1.58(1)$ & $2.77(2)$ & $0.396(5)$ & $10(7)$ & 8.87 & \\
\hline
\end{tabular}

\# Maximal or minimal expression levels observed in the non-infected or Salmonella Enteritidis infected chickens as determined in the time-dependent study; numbers in brackets indicate the day of life when the appropriate value was recorded.

\$ Maximal fold upregulation or downregulation and the day when recorded in the time-dependent study. The downregulated genes are at the bottom of the table starting with AQP8.

\& Fold up or downregulations 4 days post-infection of chickens with wild-type Salmonella Enteritidis and the SPI1 mutant.

onwards and some of the infected chickens aged 22 days or more already merged with the non-infected controls (Figure 4).

Influence of the SPI1-encoded type III secretion system of Salmonella Enteritidis on chicken gene expression in the cecum

Salmonella invasion of non-professional phagocytes is mediated by a type III secretion system encoded by Salmonella Pathogenicity Island 1 (SPI1) [16]. In the final experiment we therefore addressed to what extent the response in the chicken cecum to Salmonella Enteritidis infection depends on an intact SPI1. To test this hypothesis, we infected chickens with wild type Salmonella Enteritidis and an isogenic mutant in which the entire SPI-1 region was deleted [9]. Whilst the infection with the wild-type Salmonella Enteritidis induced expression to a similar extent as in the time-dependent experiment, the SPI1 mutant essentially did not induce an immune response in the chicken cecum (Table 1 ). Only MMP7 was significantly induced in the cecum 4 days after the infection with the Salmonella Enteritidis SPI1 mutant. However, the fold induction (35 fold) after infection with the SPI1 mutant was $45 \times$ lower when compared with the MMP7 induction in the cecum of chickens infected with the wild-type Salmonella Enteritidis (1578 fold, see Table 1). This difference could not be caused by a mere absence of the SPI1 mutant from the chicken cecum, liver or spleen, since the

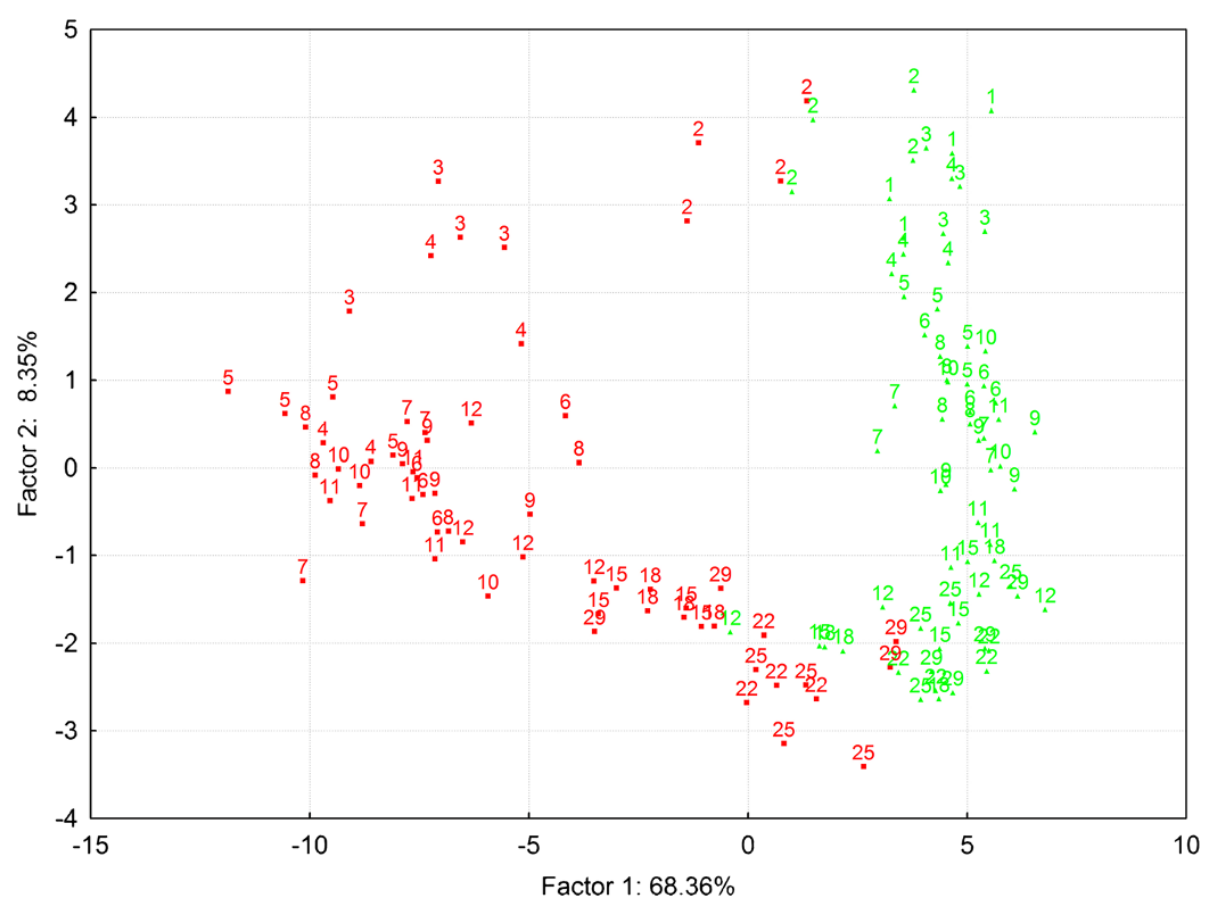

Figure 4 PCA of the chickens clustered according to their gene expression in the cecum. Sixty-eight percent of variability explained by coordinate 1 is likely to be associated with the infection status. Coordinate 2 explaining an additional $8 \%$ of variability seems to be chicken age. Green and red color, clustering of the non-infected and infected chickens, respectively. Numbers indicate age of each particular chicken in days. 
SPI1 mutant was detected in all of these tissues, though at lower counts than the wild-type Salmonella Enteritidis (Table 2).

\section{Discussion}

In this study we determined gene expression in the chicken cecum after Salmonella Enteritidis infection. Out of 9 genes downregulated after Salmonella Enteritidis infection expression of which we characterised in detail, 15hydroxyprostaglandin dehydrogenase (HPGD) might be directly involved in host defense. 15-hydroxyprostaglandin dehydrogenase is an enzyme involved in the inactivation of prostaglandin D2. Interestingly, among the genes with increased expression, prostaglandin D2 synthase (PTGDS) has been identified. An increase in the expression of an enzyme required for the biosynthesis of prostaglandin D2 together with a decreased expression of the inactivating enzyme suggest that prostaglandin D2 accumulates in the cecum of infected chickens as one of the inflammatory signals.

Among the genes upregulated after the infection, induction rates greater than 100 fold were observed for MMP7, IgG, IRG1, SAA, ExFABP, IL-22, TRAP6, MRP126, IFN $\gamma$, iNOS, ES1, IL-1ß, LYG2, IFIT5, IL-17, AVD, AH221 and SERPIN B. On the other hand, genes which exhibited the highest expression levels, irrespective of their fold increase, included AVD, ExFABP, ES1, IgG, IgA, AH221, PTGDS, SAA, MMP7, RSFR, LYG2 and TRAP6. Comparison of these two sets of genes showed that highly inducible cytokine encoding genes were absent among the genes with the highest absolute level of expression. This points to one limitation of our study. Since we purified total mRNA and/or protein from total cecal tissue, we could not detect changes in the gene expressions which may have occurred in the cells forming a minority in the gut tissue even if the change in expression levels within specific cells reached a factor of 100, as was the case of IL-17, IL-22 and IFNy expressed by T-lymphocytes [17].

Although the majority of the genes identified in this study have not been associated with the chicken response to Salmonella Enteritidis infection, they have been described as inducible during different inflammatory diseases, cell differentiation or cancer. Based on the composition of genes upregulated after the infection it is also clear that the response was a coordinated action of epithelial cells (induction of MMP7, LYG2, IL-8), T-lymphocytes (IL-1 $\beta$, IL-17,

\begin{tabular}{|c|c|c|c|}
\hline & $\begin{array}{l}\text { Liver } \\
\text { (CFU/g) }\end{array}$ & $\begin{array}{l}\text { Spleen } \\
\text { (CFU/g) }\end{array}$ & $\begin{array}{l}\text { Cecum } \\
\text { (CFU/g) }\end{array}$ \\
\hline Salmonella Enteritidis & $4.13 \pm 0.70$ & $4.31 \pm 1.80$ & $8.41 \pm 0.57$ \\
\hline Salmonella Enteritidis $\Delta$ SPI1 & $2.95 \pm 1.58$ & $2.19 \pm 1.71$ & $7.78 \pm 0.59$ \\
\hline
\end{tabular}

IL-22, IFN $\gamma$ ), macrophages and heterophils (IRG1, SAA, ExFABP, TRAP6, MRP126, iNOS, AVD, AH221, IFIT5) and B-lymphocytes (IgG, IgA).

Except for MMP7, the gene expressions characterized in this study were not triggered after infection with the noninvasive SPI1 mutant of Salmonella Enteritidis. However, even in the case of MMP7, its induction 4 days postinfection with the SPI1 mutant (35 fold) was significantly lower than after infection with wild-type Salmonella Enteritidis (1578 fold). This means that the signaling was strictly dependent on Salmonella invasion into nonprofessional phagocytes encoded by SPI1 [16] and the intracellular presence of Salmonella Enteritidis.

Two genes upregulated after infection exhibited quite a different pattern of expression from the "inflammatory" ones and these included the transcripts coding for the constant parts of IgG and IgA. As we proposed previously, the level of transcription of IgG and IgA increases in the chicken cecum with age even in the non-infected chickens [6] and infection of chickens with Salmonella Enteritidis increased the expression of IgG and IgA further. Maximal IgG and IgA expression after the infection was recorded when the expression of the remaining inflammatory genes began to decline. Interestingly, Wei et al. reported that the interaction of B-lymphocytes and CD8 T-lymphocytes was necessary for a decrease of CD4 T-lymphocyte activity at the site of epithelial inflammation and this interaction was dependent on TAP1 [18,19], another gene upregulated during the infection but declining when IgG and IgA increased in expression. TAP1 is involved in the presentation of self antigens in association with $\mathrm{MHCI}$ and increased expression of TAP1 was recorded in piglets in response to colonization with conventional microbiota [20], or in the lungs of Salmonella infected pigs [21]. This would point towards an important role of immunoglobulins or B-lymphocytes in the final recovery phase of infection. Another gene which was induced in the chicken cecum and may support the hypothesis on an important role of immunoglobulins or B-lymphocytes is HCLS1. HCLS1, also known as HS1, is expressed in all haematopoetic cells [22] and is involved in antigen-receptor signaling in both T- and B-lymphocytes. After crosslinking of the B-cell receptor, HCLS1 is phosphorylated [23] and associates with the B-cell receptor in lipid rafts of murine and chicken B-lymphoid tissue cultures [24]. Moreover, HCLS1 knockout mice are less responsive to T-cell independent antigens [25]. This suggests that the recovery phase is associated with B-lymphocytes and immunoglobulin production against T-cell independent antigens such as LPS. This is completely consistent with the use of LPS ELISA for the detection of anti-Salmonella antibodies [8]. Moreover, two groups of Crohn's disease patients have been reported, either with increased or decreased B1-like cells producing low affinity antibodies in a 
T-cell independent manner. Interestingly, patients with decreased B1-like cells were subjected to surgery more frequently than the patients with increased B1-like cells in the intestinal tract [26]. This further supports the hypothesis that the recovery phase is dependent on immunoglobulin production and that these antibodies might be targeted primarily against thymus independent antigens such as Salmonella LPS [8].

Based on our results we propose that the chicken immune response to Salmonella Enteritidis infection occurs in four steps. During the first step IL-8 and IL-17 signaling is triggered and the simplest defense mechanisms involving lysozyme G2 and type I interferon inducible protein IFIT5 are activated. This phase peaks at 2 days postinfection. The second phase is associated with the expression of genes characteristic of heterophils and macrophages, and acute phase proteins such as IRG1, SAA, ExFABP, TRAP6, MRP126, iNOS, IL-1 $\beta$, AVD or AH221. The peak of the phase 2 response is around day 4 postinfection. Between phase 1 and phase 2, MMP7 is induced allowing tissue relaxation and leukocyte influx and translocation across the intestinal epithelium. During the third phase from day 5 until day 15 post-infection, transcriptional regulators STAT1 and STAT3 are highly expressed, together with IFNy characteristic of CD4 T-lymphocyte activity. In addition, iNOS, ASL, C3 complement, EPSTI1, HCSL1 and ITGB2 (CD18) were also induced. Expression of these genes is characteristic of the development of Th1 type of immune response and control of Salmonella Enteritidis infection. For the last phase, the expression of immunoglobulins is the most characteristic and the immunoglobulins are likely targeted against T-cell independent antigens. This is in total agreement with the known role of IgA as a major effector in the mucosal immune system [27].

\section{Additional file}

Additional file 1: List of differentially expressed genes identified in this study. This Excel file contains additional information on differentially expressed genes identified in this study by 454 pyrosequencing, LC MS and real time PCR. In addition, primers used for the real time PCR are listed in this additional file as well.

Competing interests

The authors declare that they have no competing interests.

\section{Authors' contributions}

MM and IR designed experiments and wrote the manuscript. MM was responsible for pyrosequencing. KV performed real time PCRs. KS and ZZ did the mass spectrometry. $\mathrm{HH}$ and FS performed animal experiments. VB was responsible for statistical analysis. All authors read and approved the final manuscript.

\section{Acknowledgements}

$M M, K V, F S, H H, V B$ and IR have been supported by the project MZE0002716202 of the Czech Ministry of Agriculture, AdmireVet project CZ.1.05/2.1.00/01.0006-ED0006/01/01 from the Czech Ministry of Education and Czech Science Foundation project P502/12/0303. KS and ZZ have been supported the project "CEITEC - Central European Institute of Technology" (CZ.1.05/1.1.00/02.0068) from European Regional Development Fund and by Czech Grant Agency (project No. P206/12/G151). The funders had no role in the study design, data collection and analysis, decision to publish, or preparation of the manuscript. Authors also would like to thank Peter Eggenhuizen for his English language corrections.

\section{Author details}

${ }^{1}$ Veterinary Research Institute, Hudcova 70, Brno 621 00, Czech Republic. ${ }^{2}$ Core Facility - Proteomics, Central European Institute of Technology, Masaryk University, Kamenice 5, Brno 625 00, Czech Republic

Received: 27 March 2013 Accepted: 23 April 2013

Published: 20 May 2013

\section{References}

1. Anonymous: Report of the task force on zoonoses data collection on the analysis of the baseline study on the prevalence of Salmonella in holdings of laying hen flocks of Gallus gallus. EFSA J 2007, 97:1-84

2. Lahuerta A, Westrell T, Takkinen J, Boelaert F, Rizzi V, Helwigh B, Borck B, Korsgaard H, Ammon A, Makela P: Zoonoses in the European Union: origin, distribution and dynamics - the EFSA-ECDC summary report 2009 Euro Surveill 2011, 16(13): Available online: http://www.eurosurveillance.org/ ViewArticle.aspx?Articleld=19832.

3. Beal RK, Powers C, Wigley P, Barrow PA, Smith AL: Temporal dynamics of the cellular, humoral and cytokine responses in chickens during primary and secondary infection with Salmonella enterica serovar Typhimurium. Avian Pathol 2004, 33:25-33.

4. Berndt A, Wilhelm A, Jugert C, Pieper J, Sachse K, Methner U: Chicken cecum immune response to Salmonella enterica serovars of different levels of invasiveness. Infect Immun 2007, 75:5993-6007.

5. Crhanova M, Hradecka H, Faldynova M, Matulova M, Havlickova H, Sisak F, Rychlik I: Immune response of chicken gut to natural colonization by gut microflora and to Salmonella enterica serovar enteritidis infection. Infect Immun 2011, 79:2755-2763.

6. Matulova M, Rajova J, Vlasatikova L, Volf J, Stepanova H, Havlickova H, Sisak F, Rychlik I: Characterization of chicken spleen transcriptome after infection with Salmonella enterica serovar Enteritidis. PLoS One 2012, 7:e48101.

7. Raffatellu M, George MD, Akiyama Y, Hornsby MJ, Nuccio SP, Paixao TA, Butler BP, Chu H, Santos RL, Berger T, Mak TW, Tsolis RM, Bevins CL, Solnick $J$, Dandekar S, Bäumler AJ: Lipocalin-2 resistance confers an advantage to Salmonella enterica serotype Typhimurium for growth and survival in the inflamed intestine. Cell Host Microbe 2009, 5:476-486.

8. Matulova M, Havlickova H, Sisak F, Rychlik I: Vaccination of chickens with Salmonella Pathogenicity Island (SPI) 1 and SPI2 defective mutants of Salmonella enterica serovar Enteritidis. Vaccine 2012, 30:2090-2097.

9. Rychlik I, Karasova D, Sebkova A, Volf J, Sisak F, Havlickova H, Kummer V, Imre A, Szmolka A, Nagy B: Virulence potential of five major pathogenicity islands (SPI-1 to SPI-5) of Salmonella enterica serovar Enteritidis for chickens. BMC Microbiol 2009, 9:268.

10. Wisniewski JR, Zougman A, Nagaraj N, Mann M: Universal sample preparation method for proteome analysis. Nat Methods 2009, 6:359-362.

11. Wisniewski JR, Nagaraj N, Zougman A, Gnad F, Mann M: Brain phosphoproteome obtained by a FASP-based method reveals plasma membrane protein topology. J Proteome Res 2010, 9:3280-3289.

12. Hsu JL, Huang SY, Chen SH: Dimethyl multiplexed labeling combined with microcolumn separation and MS analysis for time course study in proteomics. Electrophoresis 2006, 27:3652-3660.

13. Rozen S, Skaletsky H: Primer3 on the WWW for general users and for biologist programmers. Methods Mol Biol 2000, 132:365-386.

14. Pilousova L, Rychlik I: Retron Se72 utilizes a unique strategy of the self-priming initiation of reverse transcription. Cell Mol Life Sci 2011, 68:3607-3617.

15. Stanford School of Medicine, Ashley Lab in the Department of Medicine. [http://ashleylab.stanford.edu/tools_scripts.html].

16. Kaniga K, Trollinger D, Galan JE: Identification of two targets of the type III protein secretion system encoded by the inv and spa loci of Salmonella typhimurium that have homology to the Shigella IpaD and IpaA proteins. J Bacteriol 1995, 177:7078-7085. 
17. Matulova M, Stepanova H, Sisak F, Havlickova H, Faldynova M, Kyrova K, Volf J, Rychlik I: Cytokine signaling in splenic leukocytes from vaccinated and nonvaccinated chickens after intravenous infection with Salmonella Enteritidis. PLoS One 2012, 7:e32346.

18. McPherson M, Wei B, Turovskaya O, Fujiwara D, Brewer S, Braun J: Colitis immunoregulation by CD8+ $T$ cell requires $T$ cell cytotoxicity and $B$ cell peptide antigen presentation. Am J Physiol Gastrointest Liver Physiol 2008, 295:G485-G492.

19. Wei B, McPherson M, Turovskaya O, Velazquez P, Fujiwara D, Brewer S, Braun J: Integration of B cells and CD8+ T in the protective regulation of systemic epithelial inflammation. Clin Immunol 2008, 127:303-312.

20. Chowdhury SR, King DE, Willing BP, Band MR, Beever JE, Lane AB, Loor JJ, Marini JC, Rund LA, Schook LB, Van Kessel AG, Gaskins HR: Transcriptome profiling of the small intestinal epithelium in germfree versus conventional piglets. BMC Genomics 2007, 8:215.

21. Zhao SH, Kuhar D, Lunney JK, Dawson H, Guidry C, Uthe JJ, Bearson SM, Recknor J, Nettleton D, Tuggle CK: Gene expression profiling in Salmonella Choleraesuis-infected porcine lung using a long oligonucleotide microarray. Mamm Genome 2006, 17:777-789.

22. Kitamura D, Kaneko H, Miyagoe Y, Ariyasu T, Watanabe T: Isolation and characterization of a novel human gene expressed specifically in the cells of hematopoietic lineage. Nucleic Acids Res 1989, 17:9367-9379.

23. Yamanashi Y, Okada M, Semba T, Yamori T, Umemori H, Tsunasawa S, Toyoshima K, Kitamura D, Watanabe T, Yamamoto T: Identification of HS1 protein as a major substrate of protein-tyrosine kinase(s) upon B-cell antigen receptor-mediated signaling. Proc Natl Acad Sci U S A 1993, 90:3631-3635

24. Hao JJ, Carey GB, Zhan X: Syk-mediated tyrosine phosphorylation is required for the association of hematopoietic lineage cell-specific protein 1 with lipid rafts and B cell antigen receptor signalosome complex. J Biol Chem 2004, 279:33413-33420.

25. Taniuchi I, Kitamura D, Maekawa Y, Fukuda T, Kishi H, Watanabe T: Antigenreceptor induced clonal expansion and deletion of lymphocytes are impaired in mice lacking HS1 protein, a substrate of the antigen-receptor-coupled tyrosine kinases. EMBO J 1995, 14:3664-3678.

26. Defendenti C, Sarzi-Puttini P, Grosso S, Croce A, Senesi O, Saibeni S, Bollani S, Almasio PL, Bruno S, Atzeni F: B lymphocyte intestinal homing in inflammatory bowel disease. BMC Immunol 2011, 12:71.

27. Suzuki K, Meek B, Doi Y, Muramatsu M, Chiba T, Honjo T, Fagarasan S: Aberrant expansion of segmented filamentous bacteria in IgA-deficient gut. Proc Natl Acad Sci U S A 2004, 101:1981-1986.

doi:10.1186/1297-9716-44-37

Cite this article as: Matulova et al:: Chicken innate immune response to oral infection with Salmonella enterica serovar Enteritidis. Veterinary Research 2013 44:37.

\section{Submit your next manuscript to BioMed Central and take full advantage of:}

- Convenient online submission

- Thorough peer review

- No space constraints or color figure charges

- Immediate publication on acceptance

- Inclusion in PubMed, CAS, Scopus and Google Scholar

- Research which is freely available for redistribution 